\title{
Codeword Design for Ultra-Wideband (UWB) Precoding
}

\author{
Yu-Hao Chang, Shang-Ho Tsai, Xiaoli Yu, and C.-C. Jay Kuo
}

\begin{abstract}
A precoding technique applied to symbols transmitted in an ultra-wideband (UWB) system can concentrate the signal power at the receiver for a higher data detection rate. The codeword design problem for optimal UWB precoding is investigated in this work. After the problem formulation, we examine its solution in the full-rank and the reduced-rank subspaces to obtain different tradeoffs between the detection performance and the amount of feedback messages. Furthermore, we propose two subspace selection schemes, each of which corresponds to a code design method. The resultant optimal codewords improve the performance of previous work significantly at the expense of a slightly increased amount of feedback messages. Finally, the performance of a precoded UWB system with various codewords is compared via computer simulation.
\end{abstract}

Index Terms - Ultra-wideband, UWB, precoding, time-reversal prefilter, TRP, pre-Rake, channel phase precoding, CPP.

\section{INTRODUCTION}

$\mathbf{T}$ HERE has been growing interest in applying the timereversal prefiltering (TRP) to the ultra-wideband (UWB) communication system [1], [2], which is also known as preRAKE diversity combining. The TRP transmitter prefilters the transmit data with the time-reversed order of the channel impulse response (CIR) so that the received signal power is well concentrated at the receiver. As compared with the conventional UWB system that employs tens or even hundreds of RAKE fingers at the receiver [3], the number of RAKE fingers required for symbol decoding is greatly reduced in TRP-UWB so that a low-cost UWB receiver becomes feasible.

The TRP-UWB system demands the channel information at the transmitter. It is however challenging to send back the entire channel information from the receiver to the transmitter due to the very large number of channel taps in an UWB channel [3]. A novel UWB transceiver system, called the channel-phase-precoded (CPP) UWB, was proposed in [4]

Manuscript received October 20, 2008; revised April 26, 2009 and September 17, 2009; accepted October 20, 2009. The associate editor coordinating the review of this paper and approving it for publication was A. Grant.

This work was supported by the Integrated Media Systems Center, a National Science Foundation Engineering Research Center, Cooperative Agreement No. EEC-9529152, and National Science Foundation contract No. CCF-0515358, and by the National Science Council (NSC), Taiwan, contract No. 97-2221-E-009-071-MY2. Any Opinions, findings and conclusions or recommendations expressed in this material are those of the authors and do not necessarily reflect those of the National Science Foundation or National Science Council. This work was presented in part at Globecom, San Francesco, CA, December 2006.

Y.-H. Chang is with MediaTek Inc., Hsinchu, Taiwan, R.O.C. (email:yuhao.chang@mediatek.com).

S.-H. Tsai is with the Department of Electrical Engineering, National Chiao Tung University, Hsinchu, Taiwan, R.O.C. (e-mail: shanghot@mail.nctu.edu.tw).

X. Yu, and C.-C. Jay Kuo are with the Ming Hsieh Department of Electrical Engineering, University of Southern California, Los Angeles, CA 90089-2564 USA (e-mail: xiaoliyu@usc.edu; cckuo@sipi.usc.edu).

Digital Object Identifier 10.1109/TWC.2010.01.081397 to alleviate this problem. The CPP-UWB transmitter encodes the data symbol with the reversed binary channel phase that takes values of +1 or -1 . Consequently, each antipodal phase information of carrierless UWB channel taps is represented by one bit so that the amount of feedback channel information is significantly reduced. The use of the reversed phase codeword leads to concentration of received signal power since all channel taps are coherently combined.

By exploiting the concentrated power, CPP-UWB can achieve a higher data rate by shortening the symbol interval of the transmit data while maintaining a tolerable intersymbol interference (ISI) level. Furthermore, it is possible to improve the system performance by selecting the length of the binary codeword to maximize the output signal-to-interference ratio (SIR). A fast search algorithm to determine the optimal codeword length was presented in [4]. The CPP-UWB system with codeword length optimization (CPP-UWB/CLO) can reduce the feedback amount furthermore since the optimal code length is typcially less than the CIR length. CPP-UWB is ideal for the case where the feedback channel capacity is scarce.

A general precoding framework for UWB systems, where the codeword can take any real value (which will be represented by $m$-bit data in practice), is considered in this work. The purpose of using multiple bits for each codeword element (rather than 1 bit in CPP-UWB) is to achieve a higher data detection rate at the expense of slightly higher communication and computational costs. We show that, by sending back a codeword with a few taps, the resultant precoded UWB system can achieve an excellent trade-off between performance and complexity in comparison with partial pre-RAKE (PPR) UWB [5] and CPP-UWB. In this context, the receiver, e.g., a mobile station (MS), delivers the estimated codeword to the transmitter, e.g., an access point (AP), without any precoding in the feedback channel. The AP can employ a complicated receiver structure, such as a Rake receiver, to combine signals from different paths to decode the transmitted codeword. Since the AP is able to perform the computational task, there is no extra cost demanded at the transceiver.

After the problem formulation, we first derive the codeword for a precoded UWB system that maximizes the output signal power or minimizes the mean square errors (MSE) at the decoder using the channel information. When we search the solution in the full codeword space, the associated computational complexity and feedback overhead are higher. To reduce these costs, we further explore a subspace approach [6], [7] and develop a reduced-rank precoding technique. That is, we search the optimal codeword from a suitably projected subspace. The complexity and the feedback overhead can be significantly reduced by the subspace approach. In 


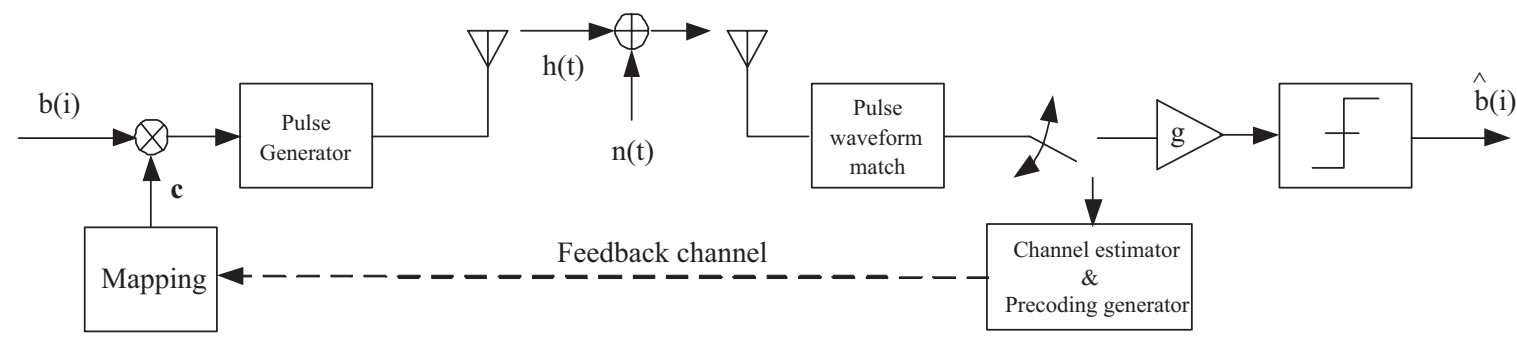

Fig. 1. The block diagram of the proposed UWB system with precoding.

general, the subspace technique demands to send back the basis vectors from the receiver to the transmitter for codeword reconstruction, which could be expensive. Here, we show that, when a proper basis set is used, the receiver only has to send the channel phase information back to the transmitter for codeword reconstruction. Thus, the additional communication burden is very low, which makes the reduced-rank precoding approach attractive. It is demonstrated by computer simulation that the reduced-rank codeword design provides an excellent tradeoff of a high data detection rate and a low communication overhead.

It is worthwhile to mention that our goal is to simplify the decoding complexity of the UWB receiver by precoding. Since only one sample per transmit bit is exploited at the receiver for symbol decoding, better system performance can be achieved if more received samples are combined at the receiver. However, such a scheme would demand a more complicated receiver architecture, such as a Rake receiver with multiple fingers. In addition, having more Rake fingers may only improve the system performance by a small margin since it is shown later that almost $78 \%$ of the total channel power is concentrated at the peak by the rank-1 codeword.

The rest of this work is organized as follows. The system model is presented in Sec. II. The full-rank and the reducedrank codeword design problems are examined in Sec. III and Sec. IV, respectively. The issue of basis selection is discussed in Sec. V. Simulation results are shown in Sec. VI. Finally, concluding remarks are given in Sec. VII.

\section{SYSTEM MODEL}

A carrierless tap-delay-line (TDL) channel model in [8] is adopted here, which can be written as

$$
h(t)=\sum_{i=0}^{L-1} h_{i} \delta(t-i \Delta)=\sum_{i=0}^{L-1} p_{i} \alpha_{i} \delta(t-i \Delta),
$$

where $h_{i}=p_{i} \alpha_{i}, L$ is the total number of resolved signal paths, $\delta(x)$ is the Dirac delta function of $x, \Delta$ is

the multipath resolution that is chosen to be the time spread of one pulse waveform (i.e., the signal pulse width), $p_{i} \in$ $\{+1,-1\}^{1}$ with equal probability is the $i$ th channel phase and $\alpha_{i}$ is the corresponding amplitude component. Parameter $\alpha_{i}$ is modelled as a Rayleigh random variable whose probability density function (PDF) is $f_{\alpha_{i}}(x)=\frac{x}{\sigma_{i}^{2}} e^{-x^{2} / 2 \sigma_{i}^{2}}$. In addition, the average power of $h_{i}$ decreases exponentially with respect

\footnotetext{
${ }^{1}$ The phase of a signal, $x$, is typically defined in complex baseband as $\arctan \{\operatorname{Im}(x) / \operatorname{Re}(x)\}$. Here, we consider the real baseband signal and view the sign of the signal as its phase information. Thus, the proposed system is restricted to the baseband system.
}

to its index $i$, i.e., $E\left\{\alpha_{i}^{2}\right\}=2 \sigma_{i}^{2}=\Omega \gamma^{i}$, where $E\{x\}$ is the expected value of random variable $x, \Omega$ is the power of the first tap and $\gamma=e^{-\Delta / \Gamma}$, and where $\Gamma$ is a decay time constant. Four different $\Gamma$ values that correspond to four suggested UWB channel models; namely, CM $1 \sim \mathrm{CM} 4$ in [9], were given in [8]. In the current context, it is assumed that the channel coherent time is long enough so that the channel remains unchanged during the transmission of one package of data symbols. It is worthwhile to point out that the equal-distance TDL channel model adopted is suitable for the indoor environment, where the multipath components are dense and the inter-arrival time is small. Thus, we can treat all propagation paths within a regular time grid as one effective channel tap [10], [11].

The block diagram of the precoded UWB system is shown in Fig.1. The $i$ th antipodal data symbol $b(i)$ with power $P$, i.e., $E\left\{b(i)^{2}\right\}=P$, is encoded by codeword $\mathbf{c}=\left[c_{0}, \cdots, c_{L-1}\right]^{T}$ and then modulated by pulse waveform $w_{s}(t)$ of unit power. Consequently, the transmit signal is of the following form

$$
x_{s}(t)=\sum_{i=-\infty}^{\infty} b(i) \sum_{j=0}^{L-1} c_{j} w_{s}\left(t-j \Delta-i T_{s}\right),
$$

where $T_{s}=M \Delta$ is the symbol interval which is assumed to be an integer multiple of the pulse width. Note that the main difference between CPP-UWB in [4] and the system in Fig. 1 is the constraint imposed on codeword $\mathbf{c}=\left[c_{0}, \cdots, c_{L-1}\right]^{T}$. Elements $c_{i}, 0 \leq i \leq L-1$, take the value of +1 or -1 in CPPUWB but they can be any real number in the current system. Thus, the current system provides a more general framework than that in [4].

After the signal is transmitted through the channel, the received signal $y(t)$ can be represented as

$$
y(t)=\sum_{l=-\infty}^{\infty} b(l) \sum_{j=0}^{L-1} \sum_{k=0}^{L-1} c_{j} h_{k} w_{r}\left(t-l T_{s}-(j+k) \Delta\right)+n(t),
$$

where $w_{r}(t)$ is the received pulse waveform of unit power and $n(t)$ is a zero mean white Gaussian noise process whose two-sided PSD is equal to $N_{0} / 2$. The receiver digitalizes the received signal by matching the pulse waveform $w_{r}(t)$, sampling the matched-filter output at the chip rate, and then amplifying the resultant digital signal by a factor of $g$. The amplifier is used to strike a balance between the channel gain and noise power suppression [12]. As a result, the matrix representation of the received signal can be written as

$$
\mathbf{r}(i)=g \mathbf{H} \mathbf{c} b(i)+g \mathbf{I}(i)+g \mathbf{n}(i),
$$

where $\mathbf{H}$ is the $(2 L-1) \times L$ Toeplitz matrix whose first column contains $\mathbf{h}=\left[h_{0}, \cdots, h_{L-1}\right]^{T}$ as the first $L$ ele- 
ments and zero afterwards, $\mathbf{r}(i)=\left[r_{0}(i), \cdots, r_{2 L-2}(i)\right]^{T}$, $\mathbf{I}(i)=\left[I_{0}(i), \cdots, I_{2 L-2}(i)\right]^{T}$ is the interference vector for transmit symbol $b(i)$, and $\mathbf{n}(i)=\left[n_{0}(i), \cdots, n_{2 L-2}(i)\right]^{T}$ is the AWGN vector for the same transmit symbol, where each element of $\mathbf{n}(i)$ is with zero mean and variance $N_{0} / 2$. Let $\mathfrak{h}=\mathbf{H c}=\left[\mathfrak{h}_{0}, \cdots, \mathfrak{h}_{2 L-2}\right]^{T}$, where

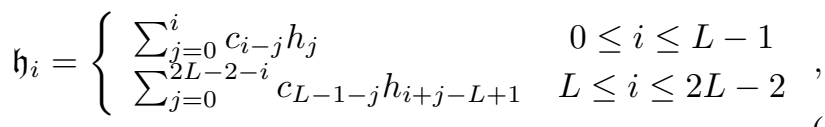

we can represent $I_{l}(i)$ and $r_{l}(i)$ explicitly as

$$
I_{l}(i)=\sum_{j=1}^{\lfloor l / M\rfloor} \mathfrak{h}_{l-j M} b(i+j)+\sum_{j=1}^{\lfloor(2 L-2-l) / M\rfloor} \mathfrak{h}_{l+j M} b(i-j)
$$

and $r_{l}(i)=g \mathfrak{h}_{l} b(i)+g I_{l}(i)+g n_{l}(i)$, where $\lfloor x\rfloor$ is the floor function of $x$. To demonstrate the performance gain due to the increase of the feedback information as compared to CPP-UWB, we adopt the same receiver structure as the CPP receiver, i.e., a single tap filter, in this work. In other words, the receiver directly applies the zero decision threshold to $r_{L-1}(i)$ to decode the $i$ th transmit symbol, i.e., $\hat{b}(i)=\operatorname{sign}\left\{r_{L-1}(i)\right\}$. Another advantage of the simple receiver structure is its low implementational complexity, which is a desirable feature for the UWB receiver.

It is worthwhile to point out that, when $T_{s} \geq L \Delta, r_{L-1}(i)$ contains no ISI. On the other hand, if $T_{s}<L \Delta, r_{L-1}(i)$ contains signals from $b(i)$ as well as $b\left(i-L_{1}\right), \cdots, b(i-1)$ and $b(i+1), \cdots, b\left(i+L_{1}\right)$ where $L_{1}=\lfloor(L-1) / M\rfloor$. As a result, the system performance degrades due to the presence of ISI at $r_{L-1}(i)$.

\section{Optimal Codeword Design}

Since only one received sample, i.e., $r_{L-1}(i)$, is used to decode the $i$ th transmit data, the receiver can reduce its sampling rate by taking one sample in every $T_{s}$ interval in a synchronized environment. Then, we can further simplify (3) to be

$$
r_{L-1}(i)=g \mathbf{c}^{T} \overline{\mathbf{H}} \mathbf{b}(i)+g n_{L-1}(i),
$$

where $\mathbf{b}(i)=\left[b\left(i+L_{1}\right), \cdots, b(i), \cdots, b\left(i-L_{1}\right)\right]^{T}$ and $\overline{\mathbf{H}}$ is a $L \times\left(2 L_{1}+1\right)$ matrix formed by transposing matrix $\mathbf{H}$ and keeping the $(L+k M)$ th column in $\mathbf{H}^{T}\left(-L_{1} \leq k \leq L_{1}\right)$, and removing all other irrelevant columns in $\mathbf{H}^{T}$. The signal part of $r_{L-1}(i)$ is $g \mathbf{c}^{T} \overline{\mathbf{h}} b(i)$, where $\overline{\mathbf{h}}=\left[h_{L-1}, \cdots, h_{0}\right]^{T}$ is the reversed channel vector.

If the CIR is known to the receiver, the receiver can compute the optimal codeword according to some criterion and send it back to the transmitter via a reliable feedback channel. In the following, we consider two criteria; namely, signal power maximization (SPM) and output mean-squared error minimization (MMSE).

\section{A. Codeword for Signal Power Maximization (SPM)}

Based on a channel realization, we can construct codeword $\mathbf{c}_{s p m}$ that maximizes the signal power at $r_{L-1}(i)$ subject to the unit-power constraint on c, i.e.,

$$
\mathbf{c}_{\text {spm }}=\arg \max _{\mathbf{c}}\left(\overline{\mathbf{h}}^{T} \mathbf{c} b(i)\right)^{2} \text { s.t. } \mathbf{c}^{T} \mathbf{c}=1 .
$$

Since the receiver gain amplifies both the signal power and the noise power at the same time, we simply set $g=1$ in (6). The solution to (7) can be easily shown as

$$
\mathbf{c}_{s p m}=\frac{\overline{\mathbf{h}}}{\|\overline{\mathbf{h}}\|},
$$

where $\|\mathbf{x}\|$ is the 2-norm of vector $\mathbf{x}$. Please note that $\mathbf{c}_{\text {spm }}$ is the unit-power reversed channel vector, i.e., the timereversal prefilter (TRP) [1] or pre-Rake [2], [5]. Although $\mathbf{c}_{s p m}$ maximizes the signal power at $r_{L-1}(i)$, it may not suppress possible ISI efficiently when $T_{s}<L \Delta$. In this case, a codeword that suppresses potential ISI while maintaining high signal power concentration is desirable.

\section{B. Codeword for Mean-Squared Error Minimization (MMSE)}

Another codeword design scheme is to minimize the meansquared error (MSE) at the receiver output [12]. According to (6), the optimal codeword, $\mathbf{c}_{m m s e}$, and the amplifier gain, $g_{m m s e}$, are chosen to minimize the value of $\varepsilon$ with unit-power c; namely,

$$
\begin{aligned}
& \left(\mathbf{c}_{m m s e}, g_{m m s e}\right) \\
& =\arg \min _{\mathbf{c}, g} E\left\{\left|b(i)-g \mathbf{c}^{T} \overline{\mathbf{H}} \mathbf{b}(i)-g n_{L-1}(i)\right|^{2} \mid \mathbf{h}\right\} \\
& \text { s.t. } \quad \mathbf{c}^{T} \mathbf{c}=1 .
\end{aligned}
$$

By applying the Lagrange multipliers to (9) [12], we get

$$
\mathbf{c}_{m m s e}=\frac{1}{g_{m m s e}}\left(\overline{\mathbf{H}} \overline{\mathbf{H}}^{T}+\frac{N_{0}}{2 P} \mathbf{I}_{L}\right)^{-1} \overline{\mathbf{h}}
$$

and

$$
g_{m m s e}=\sqrt{\overline{\mathbf{h}}^{T}\left(\overline{\mathbf{H}} \overline{\mathbf{H}}^{T}+\frac{N_{0}}{2 P} \mathbf{I}_{L}\right)^{-2} \overline{\mathbf{h}}},
$$

where $\mathbf{I}_{L}$ is the identity matrix of size $L \times L$. Although the expression of the MMSE codeword in (10) is similar to that in [12], we would like to emphasize that it is derived with a different objective here. In [12], the optimal prefilter was designed to suppress all off-peak signals. However, it may not be able to minimize the output MSE in (10). In contrast, our MMSE codeword is designed to minimize the output MSE.

It is worthwhile to emphasize that the codeword of SPM or MMSE precoding is a function of the current channel response. Therefore, both schemes are suitable when the channel is quasi-stationary. If the channel response changes, additional overhead for channel update is needed. In addition, both SPM and MMSE codeword schemes reduce the multipath combining complexity of the conventional Rake receiver at a different cost. That is, the SPM codeword scheme demands additional feedback overhead to send the estimated channel response to the transmitter while the MMSE scheme needs extra computational power to calculate the MMSE solution. However, the MMSE codeword is superior to the SPM codeword from the interference suppression prospective and the receiver can select the proper codeword based on the complexity and performance trade-off. 


\section{REDUCED-RANK CODEWORd DESIGN}

We can choose an $L \times d$ matrix, denoted by $\mathbf{M}_{d}$, whose columns are orthonormal to each other, and consider projections onto a subspace via

$$
\overline{\mathbf{h}}^{(d)}=\mathbf{M}_{d}^{T} \overline{\mathbf{h}}
$$

and

$$
\overline{\mathbf{H}}^{(d)}=\mathbf{M}_{d}^{T} \overline{\mathbf{H}},
$$

which are projections of $\overline{\mathbf{h}}$ and $\overline{\mathbf{H}}$ onto $\mathbf{M}_{d}$, respectively. Then, the reduced-rank codeword for $\mathbf{c}_{s p m}$ and $\mathbf{c}_{m m s e}$ in the subspace spanned by $\mathbf{M}_{d}$ can be written as

$$
\hat{\mathbf{c}}_{s p m}^{(d)}=\frac{\mathbf{M}_{d}^{T} \overline{\mathbf{h}}}{\left\|\mathbf{M}_{d}^{T} \overline{\mathbf{h}}\right\|}
$$

and

$$
\hat{\mathbf{c}}_{m m s e}^{(d)}=\frac{1}{\hat{g}_{m m s e}^{(d)}}\left(\overline{\mathbf{H}}^{(d)} \overline{\mathbf{H}}^{(d), T}+\frac{N_{0}}{2 P} \mathbf{I}_{d}\right)^{-1} \overline{\mathbf{h}}^{(d)},
$$

respectively, where

$$
\hat{g}_{m m s e}^{(d)}=\sqrt{\overline{\mathbf{h}}^{(d), T}\left(\overline{\mathbf{H}}^{(d)} \overline{\mathbf{H}}^{(d), T}+\frac{N_{0}}{2 P} \mathbf{I}_{d}\right)^{-2} \overline{\mathbf{h}}^{(d)},}
$$

Since the size of matrix in (15) to be inverted is only $d \times d$, its computational cost is lower than that in (10).

Once codeword $\hat{\mathbf{c}}_{s p m}^{(d)}$ or $\hat{\mathbf{c}}_{m m s e}^{(d)}$ is found, the receiver can send it back to the transmitter with an overhead lower than sending the full-rank code, i.e., $\mathbf{c}_{\text {spm }}$ or $\mathbf{c}_{m m s e}$. After receiving $d$ coefficients $\hat{\mathbf{c}}_{s p m}^{(d)}$ or $\hat{\mathbf{c}}_{m m s e}^{(d)}$, the transmitter can synthesize the $L \times 1$ codeword $\mathbf{c}_{s p m}^{(d)}$ or $\mathbf{c}_{m m s e}^{(d)}$ by

$$
\mathbf{c}_{s p m}^{(d)}=\mathbf{M}_{d} \hat{\mathbf{c}}_{s p m}^{(d)} \quad \text { and } \quad \mathbf{c}_{m m s e}^{(d)}=\mathbf{M}_{d} \hat{\mathbf{c}}_{m m s e}^{(d)} .
$$

It can be shown easily that the peak power $P_{s p m}^{(d)}$ generated by $\mathbf{c}_{s p m}^{(d)}$ and the output MSE achieved by $\mathbf{c}_{m m s e}^{(d)}$ are

$$
P_{\text {spm }}^{(d)}=P\left\|\mathbf{M}_{d}^{T} \overline{\mathbf{h}}\right\|^{2}
$$

and

$$
\varepsilon_{\text {min }}^{(d)}=P\left(1-\overline{\mathbf{h}}^{(d), T}\left(\overline{\mathbf{H}}^{(d)} \overline{\mathbf{H}}^{(d), T}+\frac{N_{0}}{2 P} \mathbf{I}_{d}\right)^{-1} \overline{\mathbf{h}}^{(d)}\right) .
$$

Codeword $\mathbf{c}_{s p m}^{(d)}$ and $\mathbf{c}_{m m s e}^{(d)}$ are truly optimal in terms of maximizing the signal power and minimizing the output MSE, respectively, for a subspace spanned by $\mathbf{M}_{d}$. However, as compared to the full-rank code, i.e., $\mathbf{c}_{s p m}$ and $\mathbf{c}_{m m s e}$, they are only suboptimal since some useful signal power is dropped by subspace filtering. Furthermore, both the transmitter and the receiver need the information of $\mathbf{M}_{d}$. It is in general costly to send back the basis information.

In the following, we will describe a simple method where the transmitter and the receiver can compute basis vectors for projection matrix $\mathbf{M}_{d}$ individually with a small amount of feedback information. The reversed channel vector $\overline{\mathbf{h}}$ can be expressed as

$$
\overline{\mathbf{h}}=\overline{\mathbf{P}} \overline{\mathbf{a}},
$$

where $\overline{\mathbf{P}}=\operatorname{diag}\left[p_{L-1}, \cdots, p_{0}\right]$ is a diagonal matrix whose $i$ th diagonal element is the phase of the $(L-i)$ th path that takes values of +1 or -1 and $\overline{\mathbf{a}}=\left[\alpha_{L-1}, \cdots, \alpha_{0}\right]^{T}$ is the reversed order of the amplitude vector. The autocorrelation of $\overline{\mathbf{a}}$ can be found by

$$
\mathbf{R}_{\overline{\mathbf{a}}}=E\left\{\overline{\mathbf{a}}^{T}\right\}=\Omega\left[\begin{array}{cccc}
\beta^{2(L-1)} & \alpha \beta^{2 L-3} & \cdots & \alpha \beta^{L-1} \\
\alpha \beta^{2 L-3} & \beta^{2(L-2)} & \cdots & \alpha \beta^{L-2} \\
\vdots & \vdots & \ddots & \vdots \\
\alpha \beta^{L-1} & \alpha \beta^{L-2} & \cdots & 1
\end{array}\right],
$$

where $\alpha=\pi / 4$ and $\beta=\gamma^{1 / 2}=e^{-\Delta / 2 \Gamma}$. The eigendecomposition of $\mathbf{R}_{\overline{\mathbf{a}}}$ can be written as

$$
\mathbf{R}_{\overline{\mathbf{a}}}=\mathbf{E}_{\overline{\mathbf{a}}} \boldsymbol{\Lambda}_{\overline{\mathbf{a}}} \mathbf{E}_{\overline{\mathbf{a}}}^{T},
$$

where $\boldsymbol{\Lambda}_{\overline{\mathbf{a}}}=\operatorname{diga}\left[\lambda_{0}, \cdots, \lambda_{L-1}\right]$ is the diagonal matrix of $L$ eigenvalues with $\lambda_{0} \geq \cdots \geq \lambda_{L-1}$, and $\mathbf{E}_{\overline{\mathbf{a}}}=\left[\tilde{\mathbf{e}}_{0}, \cdots, \tilde{\mathbf{e}}_{L-1}\right]$ is a matrix whose columns are the corresponding eigenvectors.

A set of full-rank basis vectors can be written as

$$
\mathbf{M}=\left[\mathbf{e}_{0}, \cdots, \mathbf{e}_{L-1}\right]=\overline{\mathbf{P}} \mathbf{E}_{\overline{\mathbf{a}}}=\left[\overline{\mathbf{P}} \tilde{\mathbf{e}}_{0}, \cdots, \overline{\mathbf{P}} \tilde{\mathbf{e}}_{L-1}\right],
$$

where $\mathbf{e}_{m}=\overline{\mathbf{P}} \tilde{\mathbf{e}}_{m}, 0 \leq m \leq L-1$. The desired projection matrix $\mathbf{M}_{d}$ is formed by collecting $d$ columns from $\mathbf{M}$, i.e., $\mathbf{M}_{d}=\left[\mathbf{e}_{i_{0}}, \cdots, \mathbf{e}_{i_{d-1}}\right]$, where $0 \leq i_{0}<\cdots<i_{d-1} \leq L-1$.

It is worthwhile to comment that, although a simplified channel model is used to illustrate the proposed reduced-rank precoding scheme, our method can be applied to other channel models as long as their second-order statistics is available at both ends of the link. We will study the performance of the proposed precoding scheme with a more practical channel model as proposed in [13] in the simulation section. In addition, since autocorrelation matrix $\mathbf{R}_{\overline{\mathbf{a}}}$ is a function of $\Gamma$ and $\Delta$ only, the eigen-decomposition of $\mathbf{R}_{\overline{\mathbf{a}}}$ in (22) can be computed off-line. The selection of $d$ from $L$ basis vectors will be detailed in the next section.

In this work, we do not attempt to quantify the value of $d$ as a function of $L$ since their relationship is a non-linear one. Instead, their relationship will be determined by the simulation shown in Sec. VI.

\section{BASIS SElection Algorithms}

The choice of proper $d$ basis vectors, $\mathbf{e}_{i_{0}}, \cdots, \mathbf{e}_{i_{d-1}}$, for reduced-rank codeword design plays an important role in the system performance. In this section, we consider two selection algorithms.

\section{A. Basis Selection for SPM Codeword}

The following proposition provides a basis selection algorithm for the construction of the SPM codeword $\mathbf{c}_{s p m}^{(d)}$ in a $d$-dimensional subspace.

Proposition 1: We can maximize the average signal power at $r_{L-1}(i)$ by constructing projection matrix $\mathbf{M}_{d}$ using the first $d$ column vectors in $\mathbf{M}$.

Proof: By substituting $\mathbf{M}_{d}=\overline{\mathbf{P}} \tilde{\mathbf{M}}_{d}$ and $\tilde{\mathbf{M}}_{d}=\left[\tilde{\mathbf{e}}_{i_{0}}, \cdots, \tilde{\mathbf{e}}_{i_{d-1}}\right]$ into (18), we can further simplify (18) to

$$
P_{s p m}^{(d)}=P\left(\overline{\mathbf{a}}^{T} \tilde{\mathbf{M}}_{d} \tilde{\mathbf{M}}_{d}^{T} \overline{\mathbf{a}}\right) .
$$


The average value of $P_{s p m}^{(d)}$ can be calculated as

$$
\begin{aligned}
\bar{P}_{s p m}^{(d)} & =E\left\{P_{s p m}^{(d)}\right\}=E\left\{P\left(\overline{\mathbf{a}}^{T} \tilde{\mathbf{M}}_{d} \tilde{\mathbf{M}}_{d}^{T} \overline{\mathbf{a}}\right)\right\} \\
& =P \operatorname{tr}\left\{E\left\{\tilde{\mathbf{M}}_{d}^{T} \overline{\mathbf{a}}^{T} \tilde{\mathbf{M}}_{d}\right\}\right\}=\operatorname{Ptr}\left\{\tilde{\mathbf{M}}_{d}^{T} \mathbf{R}_{\overline{\mathbf{a}}} \tilde{\mathbf{M}}_{d}\right\} \\
& =P \sum_{m=0}^{d-1} \lambda_{i_{m}} .
\end{aligned}
$$

Since $\lambda_{0} \geq \cdots \geq \lambda_{L-1}$, we can select $i_{m}=m, 0 \leq m \leq$ $(d-1)$, to maximize $\bar{P}_{s p m}^{(d)}$.

By Proposition 1, we obtain the optimal codeword $\mathbf{c}_{s p m}^{(d)}$ in the subspace spanned by the first $d$ columns of M. It is interesting to compare the system performance with another basis set. In the following proposition, we consider the standard basis, which is formed by some $d$ columns from the $L \times L$ identity matrix.

Proposition 2: When the standard basis is used in subspace filtering to compute $\mathbf{c}_{s p m}^{(d)}$, the average signal power at $r_{L-1}(i)$ is not greater than

$$
P \sum_{m=L-d}^{L-1} \mu_{m},
$$

where $\mu_{m}$ is the average power of the $m$ th element in $\overline{\mathbf{h}}$, i.e., $\mu_{m}=E\left\{h_{L-1-m}^{2}\right\}=\Omega \gamma^{L-1-m}$. In other words, (26) is the upper bound for the average signal power at $r_{L-1}(i)$.

Proof: The proof is similar to that in Proposition 1 and thus omitted here.

To compare the signal power accumulated by these two basis sets in Proposition 3, we need the following lemma.

Lemma 1: The eigenvalues of $\mathbf{R}_{\overline{\mathbf{a}}}$ are bounded by

$$
\left\{\begin{array}{l}
\lambda_{0}>\Omega \\
\lambda_{i}<\Omega \beta^{2 i}
\end{array} \text { for } 1 \leq i \leq(L-1) .\right.
$$

Proof: The proof is given in Appendix VIII-A.

Proposition 3: If the codeword dimension $d$ is less than $L$, the basis set given in Proposition 1 generates a higher peak power at $r_{L-1}(i)$ than the standard basis set.

Proof: The proof is given in Appendix VIII-B.

Actually, the first basis vector $\mathbf{e}_{0}$ in (23) contributes much more signal power than all other basis vectors, which can be characterized by the following proposition.

Proposition 4: The signal power generated by $\mathbf{c}_{s p m}^{(1)}$ accounts for about $78 \%$ of the total power spread in the channel as $\Delta$ becomes infinitesimal. Furthermore, we have $\tilde{\mathbf{e}}_{0} \approx \frac{\mathbf{b}}{\|\mid \mathbf{b}\|}$, where b is defined in (36).

Proof: The proof is given in Appendix VIII-C.

It is worthwhile to point out that Proposition 4 is derived under the assumption that $\Delta$ is very small as compared with the decay time constant $\Gamma$. As we vary the value of $\Gamma$ to represent a different channel mode [8] (e.g., a smaller value of $\Gamma$ for the line-of-sight (LOS) channel and a larger value of $\Gamma$ for the non-LOS channel), a great number of multipath components can always be resolved and Proposition 4 still holds.

Finally, we would like to point out the relationship between the standard basis and the partial pre-RAKE (PPR) precoding method in [5]. PPR reduces the feedback overhead of conventional TRP since only the first $d$ channel taps in $\mathbf{h}$ are fed back to form parital TRP. It is easy to see that, in order to achieve the upper bound of the signal power in Proposition 2 using the standard basis set, the last $d$ standard bases must be selected. Consequently, the $d$-dimensional codeword $\mathbf{c}_{s p m}^{(d)}$ becomes the normalized version of $\left[h_{d-1}, \cdots, h_{0}\right]^{T}$, which is exactly the same as PPR. To distinguish SPM codewords constructed with different basis sets (i.e., basis set $\mathbf{M}$ introduced in (23) and the standard basis set), a SPM codeword using the last $d$ standard basis vector is called the rank- $d$ PPR codeword while a SPM codeword using the $d$ leading bases in $\mathbf{M}$ is called the rank- $d$ SPM codeword.

\section{B. Basis Selection for MMSE Codeword}

Next, we discuss basis selection to compute reduced-rank MMSE codeword $\mathbf{c}_{m m s e}^{(d)}$. Generally speaking, the optimal $d$ dimensional signal subspace that minimizes the output MSE in $\mathbf{M}_{d}$ as shown in (19) demands an exhaustive search among all possible subspaces whose cardinality is equal to $C_{d}^{L}=\frac{L !}{d !(L-d) !}$. Since $L$ is usually a large number, the associated computational cost of exhaustive search is too high to be practically useful. A suboptimal basis selection algorithm, which utilizes a sequential greedy search algorithm to determine $\mathbf{M}_{d}$, is proposed below.

To acquire the first basis vector, we search all possible basis vectors and pick up the one that provides the minimum MSE, i.e.,

$$
i_{0}=\arg \min _{i \in \mathcal{I}_{0}} P\left(1-a^{(i)}\left(\left\|\mathbf{f}^{(i)}\right\|^{2}+\frac{N_{0}}{2 P}\right)^{-1} a^{(i)}\right),
$$

where $\mathcal{I}_{0}=\{0, \cdots, L-1\}, a^{(i)}=\mathbf{e}_{i}^{T} \overline{\mathbf{h}}$, and $\mathbf{f}^{(i)}=\overline{\mathbf{H}}^{T} \mathbf{e}_{i}$. Next, we assume that $m(m<d)$ basis vectors are already selected and $\mathbf{M}_{m}=\left[\mathbf{e}_{i_{0}}, \cdots, \mathbf{e}_{i_{m-1}}\right]$. Let $\mathbf{e}_{i_{m}}$ denote the next basis vector to add so that the corresponding basis set is $\mathbf{M}_{m+1}=\left[\mathbf{M}_{m}, \mathbf{e}_{i_{m}}\right]$. The MSE in the $(m+1)$-dimensional subspace is shown in (29). By the block matrix inversion formula [14], (29) can be further simplified as

$$
\varepsilon_{\text {min }}^{(m+1)}=\varepsilon_{\text {min }}^{(m)}-P C_{i_{m}} \zeta_{i_{m}},
$$

where the values of $C_{i_{m}}$ and $\zeta_{i_{m}}$ are shown on the next page. It can be easily shown that both $C_{i_{m}}$ and $\zeta_{i_{m}}$ are non-negative, and $P C_{i_{m}} \zeta_{i_{m}}$ can be viewed as the gain due to the addition of one more basis vector $\mathbf{e}_{i_{m}}$. Hence, to minimize $\varepsilon_{\text {min }}^{(m+1)}$, the $(m+1)$ th basis vector can be chosen from the remaining index set such that the product of $C_{i_{m}}$ and $\zeta_{i_{m}}$ is maximized, i.e.,

$$
i_{m}=\arg \max _{i \in \mathcal{I}_{m}} C_{i} \zeta_{i},
$$

where $\mathcal{I}_{m}$ is the remaining index set by eliminating selected indices $\left\{i_{0}, \cdots, i_{m-1}\right\}$. By following the above procedure, we can get one more basis vector each time until the complete set of $d$ basis vectors is selected.

For a very low SNR channel, we have the following special case.

Proposition 5: When the channel SNR is asymptotically low, we can minimize the average MSE by taking the first $d$ columns from M. In other words, we choose the same subspace that maximizes the average signal power at the output of the receiver.

Proof: As the channel SNR becomes asymptotically low, the 


$$
\begin{aligned}
\varepsilon_{m i n}^{(m+1)}= & P\left(1-\left(\mathbf{M}_{m+1}^{T} \overline{\mathbf{h}}\right)^{T}\left(\left(\mathbf{M}_{m+1}^{T} \overline{\mathbf{H}}\right)\left(\mathbf{M}_{m+1}^{T} \overline{\mathbf{H}}\right)^{T}+\frac{N_{0}}{2 P} \mathbf{I}_{m+1}\right)^{-1}\left(\mathbf{M}_{m+1}^{T} \overline{\mathbf{h}}\right)\right) \\
= & P\left(1-\left[\begin{array}{c}
\overline{\mathbf{h}}^{(m)} \\
a^{\left(i_{m}\right)}
\end{array}\right]^{T}\left[\begin{array}{c|c}
\overline{\mathbf{H}}^{(m)} \overline{\mathbf{H}}^{(m), T}+\frac{N_{0}}{2 P} \mathbf{I}_{m} & \overline{\mathbf{H}}^{(m)} \mathbf{f}^{\left(i_{m}\right)} \\
\hline \mathbf{f}^{\left(i_{m}\right), T} \overline{\mathbf{H}}^{(m), T} & \left\|\mathbf{f}^{\left(i_{m}\right)}\right\|^{2}+\frac{N_{0}}{2 P}
\end{array}\right]^{-1}\left[\begin{array}{c}
\overline{\mathbf{h}}^{(m)} \\
a^{\left(i_{m}\right)}
\end{array}\right]\right) \\
C_{i_{m}}= & \left(\left\|\mathbf{f}^{\left(i_{m}\right)}\right\|^{2}+\frac{N_{0}}{2 P}-\mathbf{f}^{\left(i_{m}\right), T} \overline{\mathbf{H}}^{(m), T}\left(\overline{\mathbf{H}}^{(m)} \overline{\mathbf{H}}^{(m), T}+\frac{N_{0}}{2 P} \mathbf{I}_{m}\right)^{-1} \overline{\mathbf{H}}^{(m)} \mathbf{f}^{\left(i_{m}\right)}\right)^{-1} \\
& \zeta_{i_{m}}=\left(\mathbf{f}^{\left(i_{m}\right), T} \overline{\mathbf{H}}^{(m), T}\left(\overline{\mathbf{H}}^{(m)} \overline{\mathbf{H}}^{(m), T}+\left(N_{0} / 2 P\right) \mathbf{I}_{m}\right)^{-1} \overline{\mathbf{h}}^{(m)}-a^{\left(i_{m}\right)}\right)^{2}
\end{aligned}
$$

MMSE codeword in (15) converges to the normalized version of $\overline{\mathbf{h}}_{d}$ so that the associated MMSE value becomes

$$
\begin{aligned}
\varepsilon_{\text {min }}^{(d)} & \simeq P\left(1-\frac{2 P}{N_{0}} \overline{\mathbf{h}}_{d}^{T} \overline{\mathbf{h}}_{d}^{-}\right)=P-\frac{2 P}{N_{0}}\left(P \overline{\mathbf{h}}^{T} \mathbf{M}_{d} \mathbf{M}_{d}^{T} \overline{\mathbf{h}}\right) \\
& =P-\frac{2 P}{N_{0}} P_{s p m}^{(d)},
\end{aligned}
$$

where $P_{s p m}^{(d)}$ as defined in (18) is the signal power at the output of the receiver when $\mathbf{c}_{s p m}^{(d)}$ is appied. By averaging $\varepsilon_{\min }^{(d)}$ over all possible channel realizations, we get the averaged MSE as

$$
\bar{\varepsilon}_{\min }^{(d)}=E\left\{\varepsilon_{\min }^{(d)}\right\} \simeq P-\frac{2 P}{N_{0}} \bar{P}_{s p m}^{(d)} .
$$

We know from Proposition 1 that the maximum value of $\bar{P}_{\text {spm }}^{(d)}$ occurs when the frist $d$ columns of $\mathbf{M}$ are employed for subspace filtering in constructing the SPM codeword. Since the signal power $P$ is fixed, the reduced-rank minimum MSE must occur in the subspace formed by the first $d$ columns of M.

\section{Simulation Results}

In this section, we compare the performance of a precoded UWB system with different codewords obtained by different design criteria. The channel parameters are chosen to be $\Delta=0.7 \mathrm{~ns}, \Gamma=10 \mathrm{~ns}(\mathrm{CM} 2)$ and $L=84$ and the average channel power is normalized to unity by setting $\Omega=\frac{1-\gamma}{1-\gamma^{L}}$. Two different data rates (namely, $102 \mathrm{Mbps}$ and $204 \mathrm{Mbps}$, which corresponds to $T_{s}=9.8$ and $4.9 \mathrm{~ns}$, respectively) are used to test the performance of SPM and MMSE codewords under different ISI levels. The tradeoff between the amount of feedback messages and the number of quantization bits for each codeword element is studied. Then, we test the robustness of the proposed precoding schemes in the presence of inaccurate channel estimates. Finally, we demonstrate how to apply our precoding scheme to a more realistic channel model proposed in [13]. All simulation results presented are obtained as using 1000 independent channel realizations.

Example 1: Peak power comparison between PPR and SPM codewords.

In Example 1, we verify Proposition 3 by comparing the peak signal power using the SPM and the PPR codewords [5], which are plotted as a function of the subspace rank of codewords in Fig. 2. As shown in the figure, there is an

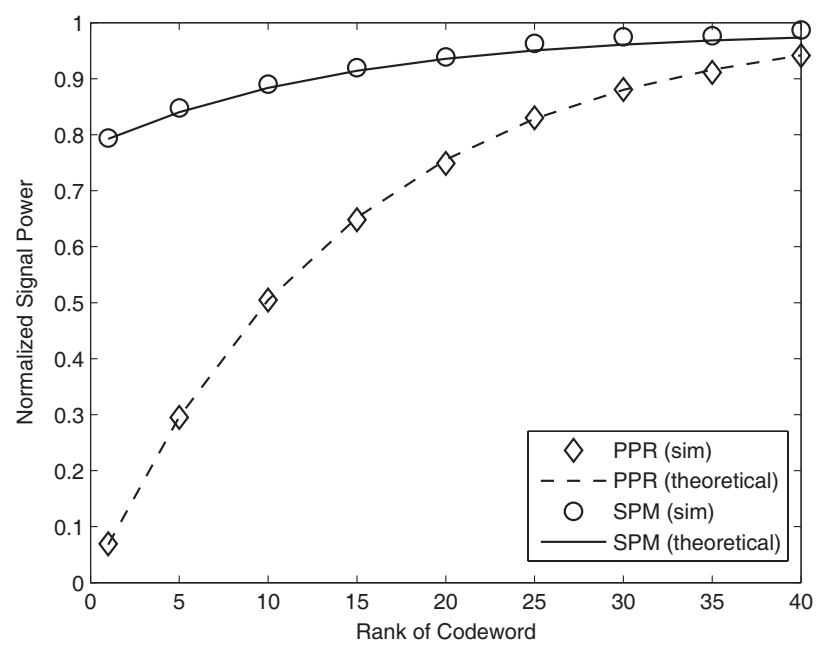

Fig. 2. Comparison of the average peak power as a function of the subspace dimension for reduced-rank PPR and SPM codewords.

excellent match of theoretical and simulation results for both codewords. Furthermore, we see that the reduced-rank SPM codeword with properly selected basis vectors as specified in Proposition 1 always has a higher signal power than the PPR codeword of the same rank. Both peaks increase as the subspace rank goes up, and the performance gap becomes smaller as the codeword rank goes higher. They eventual converge to the same level as they reach the full rank. This can be explained by Lemma 1 since the signal power is related to the cumulative sum of eigenvalues. This example demonstrates the clear advantage of SPM codewords of lower rank.

\section{Example 2: BER performance comparison for PPR and} SPM codewords.

We compare the performance of a precoded UWB system in terms of the bit error rate (BER) using SPM and PPR codewords in Example 2. The symbol interval $T_{s}$ is chosen to be $9.8 \mathrm{~ns}$, which corresponds to a data rate of $102 \mathrm{Mbps}$, and two codeword ranks, i.e. 1 and 5, are considered. We show simulation results in Fig. 3, where the BER curves of CPP-UBW/CLO, full-rank PPR (i.e., time-reversal prefilter [1]) and full-rank MMSE [12] are also shown as performance benchmarks.

We see a large performance gap between SPM and PPR at the same rank, and the BER improvement due to the increase 


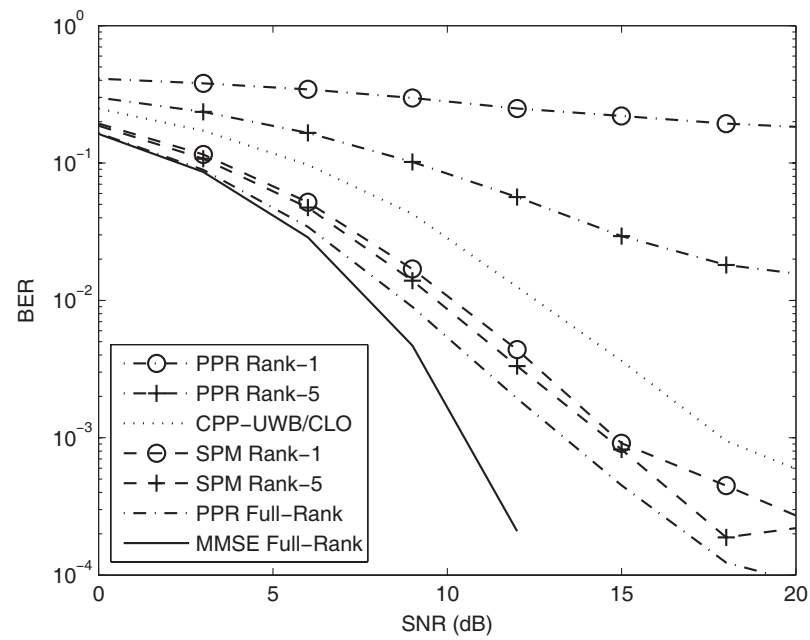

Fig. 3. The bit error rate as a function of the SNR value for different reduced-rank PPR and SPM codewords with $T_{s}=9.8 \mathrm{~ns}$ (or $102 \mathrm{Mbps}$ ).

of the codeword rank is smaller in SPM, which is consistent with our observations in Example 1. When compared with CPP-UWB/CLO, SPM improves the BER performance by increasing the communication overhead slightly.

\section{Example 3: BER performance comparison for SPM and MMSE codewords.}

In Example 3, we study the effect of higher data rates on the performance of different codeword design schemes by halving the symbol interval and keeping all other parameters the same as before. We examine the BER performance of the full-rank SPM (i.e., time-reversal prefilter [1]), which provides the performance bound on all reduced-rank SPM codewords, in Fig. 4. We also compare the BER curves of the reducedrank MMSE codeword with three different basis sets in the same figure; namely, the optimal subspace and two subspaces discussed in Subsections V-A and V-B. They are denoted by Opt, $\mathrm{A}$ and $\mathrm{B}$ in the legend, respectively. Please note that the performance curve of the optimal Rank-5 MMSE codeword is ignored since the associated complexity for the signal basis search is too high to be possible. Finally, the BER curves of full-rank MMSE and CPP-UWB/CLO are also plotted for performance comparison.

We see from Fig. 4 that the SPM codeword, which fills in the performance gap between the CPP-UWB/CLO codeword and the full-rank MMSE codeword in Example 2, cannot maintain a satisfactory performance for a higher data rate due to the presence of more ISI. By considering the ISI suppression effect in the codeword design, we can effectively reduce the detection error probability if both the filter rank and signal basis are carefully selected. In addition, the BER performance of the reduced-rank MMSE codeword can be improved by carefully choosing the basis by comparing curves with labels "A" and "B". It is worthwhile to point out that although the subspace selection scheme developed in Subsection V-B is suboptimal, it leads to almost the same decoding performance as the Rank-3 MMSE codeword in the optimal subspace while enjoying a much reduced computational complexity in basis selection. Basis selection scheme A is meant for the SPM codeword while basis selection scheme B is designed for the MMSE codeword. In Examples 4 and 5, we will consider only basis selection scheme B for the MMSE codeword.

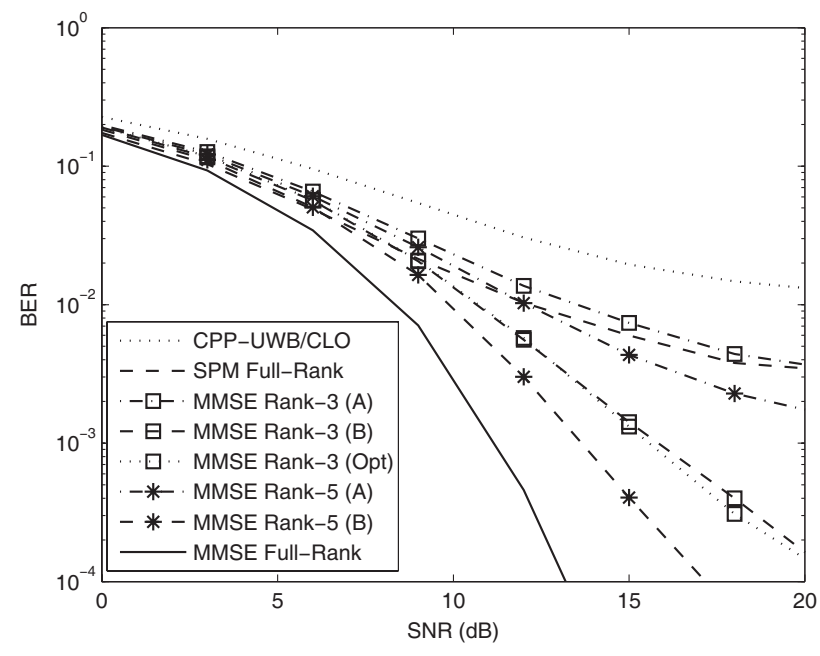

Fig. 4. The bit error rate as a function of the SNR value for different reduced-rank PPR and SPM codewords with $T_{s}=4.9 \mathrm{~ns}$ (or $204 \mathrm{Mbps}$ ), where Opt, A and B in the legend denote the optimal signal subspace and basis selection algorithms given in Sec. V-A and Sec. V-B, respectively.

\section{Example 4: BER performance of quantized MMSE code- words.}

In Examples 1-3, the transmitter can get nearly perfect codewords from the receiver in the sense that each element of the codeword is represented by a sufficient number of bits and the quantization error is small enough to ignore. Since the feedback overhead is proportional to the number of quantization bits, we study the quantization effect of codeword elements in this example. Typically, we can assume that the codeword resolution is still sufficiently high when being calculated in the receiver. Then, each codeword element is quantized to be sent back to the transmitter in order to reduce the feedback overhead. In this example, we consider 2-bit, 3-bit, 4-bit quantization per element for rank-3 and rank-5 MMSE codewords. The dynamic range is set as $\{+1,-1\}$, where 1 is the normalized channel power. The BER curves of these methods are shown in Fig. 5. The BER curves of ideal reduced-rank codewords are also shown for comparison. We see that reduced-rank codewords converge to the ideal performance with 4-bit quantization in both cases. Thus, the overhead of sending back quantized codewords from the receiver to the transmitter for precoding is extremely low. They are equal to $4 \times 3=12$ bits and $4 \times 5=20$ bits for rank- 3 and rank-5, respectively.

\section{Example 5: BER performance of codewords with imperfect channel information.}

The accuracy of the UWB channel estimates at the receiver has an effect on the codeword calculation and, hence, the overall performance of the precoded UWB system. It is difficult to get accurate channel estimation especially when there are a large number of unknown taps such as in the case of UWB channels [15]. We have so far assumed the availability of perfect channel estimation. In this example, we would like to test the robustness of reduced-rank MMSE codewords with imperfect channel estimation information. Please note that this is a topic that cannot be well treated in this work. Our preliminary study here is only of exploratory nature.

The channel information is estimated by averaging over all channel responses from $n$ training pulses and the interval 


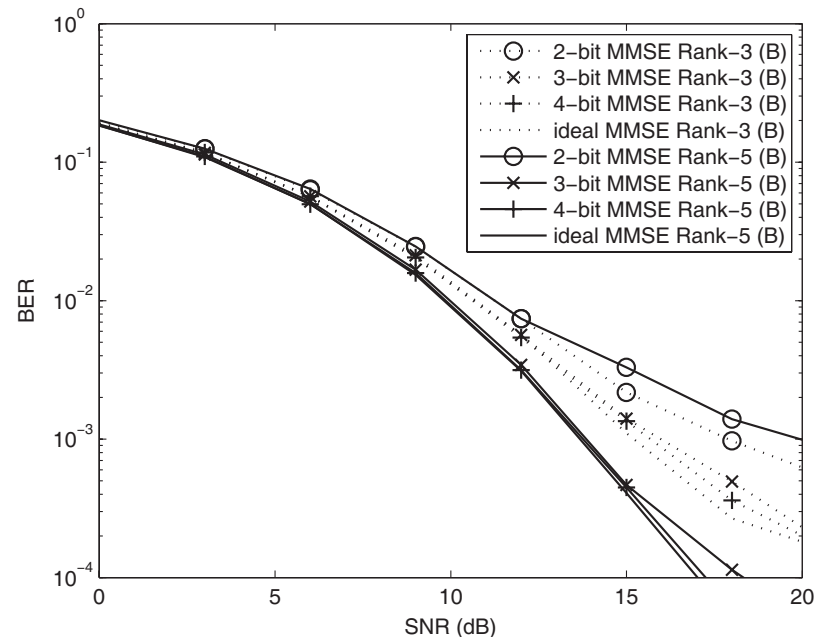

Fig. 5. The bit error rate as a function of the SNR value for reduced-rank PPR and SPM codewords quantized by a different number of bits.

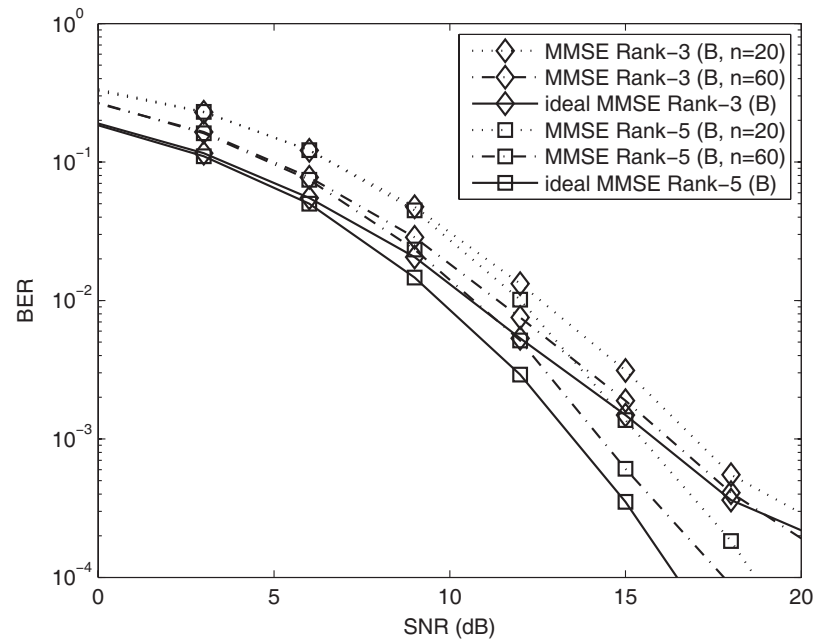

Fig. 6. The bit error rate as a function of the SNR value for reduced-rank MMSE codewords calculated based on the imperfect channel estimates.

between two consecutive pulses is properly selected to avoid ISI. The accuracy of channel estimation can be improved by increasing the number of training symbols. We consider two different cases, namely, $n=20$ and 60 , to highlight the trade-off between performance and training overhead for the different codeword rank ${ }^{2}$. Other system parameters remain the same as those in the previous example. The simulation result is drawn in Fig. 6. Again, the system performance, which corresponds to the ideal channel information at the receiver, is also plotted as the performance benchmark.

It is observed from Fig. 6 that a better BER performance can be achieved by increasing the number of training symbols for a fixed rank codeword. However, the performance of the rank-3 code converges more quickly to the ideal case than that of the rank- 5 code. This implies that a higher rank code provides a better performance gain than a lower rank code at the cost of more accurate channel knowledge, i.e., more

\footnotetext{
${ }^{2}$ The UWB communication system focuses on the application in the indoor channel, where the transmitter, receiver and the surrounding objects are almost still. In this case, the channel coherent time is long enough to cover the time duration required for training and channel information feedback.
}

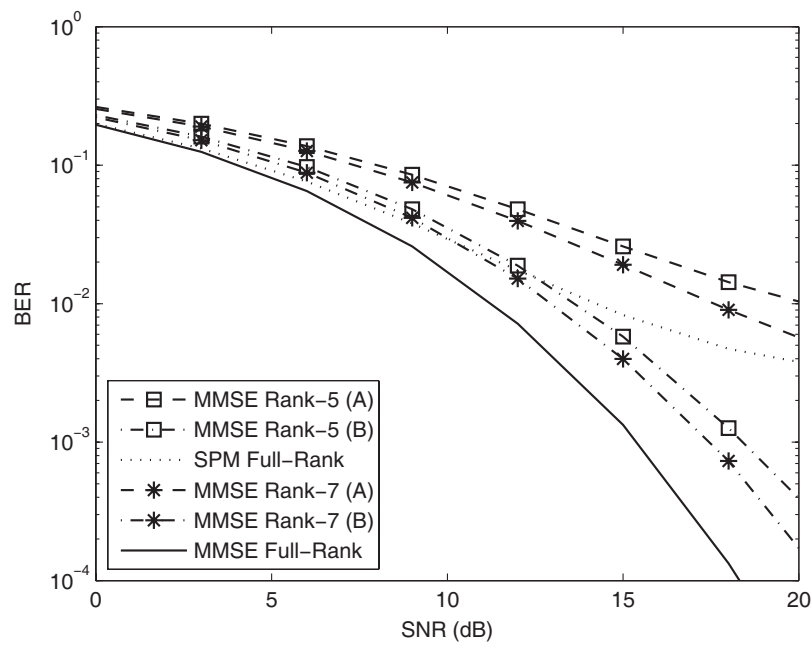

Fig. 7. The bit error rate as a function of the SNR value for different reducedrank PPR and SPM codewords under the channel model in [13], where A and $\mathrm{B}$ in the legend denote basis selection algorithms given in Subsections V-A and $\mathrm{V}-\mathrm{B}$, respectively.

training overhead in our case.

Example 6: BER performance comparison for SPM and MMSE codewords with a realistic channel model.

In the last example, we show the application of the proposed precoding scheme to a more realistic UWB channel model. A statistical channel model proposed by Foerster et al. in [13] is adopted here. The multipath resolution is fixed at $0.7 \mathrm{~ns}$ and the symbol interval is set to $4.9 \mathrm{~ns}$, which corresponds to $M=7$. In our simulation, the channel is realized based on the parameters of CM2 as specified in [13]. Note that the channel response is truncated after 84 taps since the power at the tail is small enough to be ignored. Due to the complex channel model in nature, it is not feasible to provide an analytical formula to synthesize the autocorrelation matrix of $\overline{\mathbf{a}}$. Instead, the knowledge of matrix $\mathbf{R}_{\overline{\mathbf{a}}}$ is acquired by averaging over 100000 independent channel realizations ${ }^{3}$. In addition, since our goal is to compare the BER performance of SPM and MMSE codewords of different ranks at various noise power levels, the log-normal shadowing effect is ignored for ease of implementation.

The BER curves of SPM and MMSE codewords at different rank are shown in Fig. 7, where $A$ and $B$ in the legend denote different basis selection schemes presented in Sec. V-A and Sec. V-B, respectively. We see from Fig. 7 that a lower rank MMSE codeword with carefully selected basis can outperform a higher rank MMSE codeword with poor basis selection. In fact, the advantage of MMSE over TRP would be compromised if we project the signal onto a wrong subspace.

\section{CONCLUSION}

An optimal precoding technique for the UWB system was examined in this work, which is a generalization of the binary codeword in the previously proposed CPP-UWB system to a codeword consisting more bits per element. Two codeword construction criteria were studied and methods to compute

${ }^{3}$ In practice, four different $\mathbf{R}_{\overline{\mathbf{a}}}$ matrices, which correspond to CM 1-4, can be numerically computed off-line based on four different channel models in [13]. Then, we can decompose $\mathbf{R}_{\overline{\mathbf{a}}}$ to acquire its eigenvectors and store the basis information at both ends of the communication link. 
full rank optimal codewords (of dimension $L$ ) were developed accordingly. The computation and transmission of full-rank codewords are however costly, which motivated us to search for suboptimal codewords using a reduced rank approach. Two basis selection algorithms, each of which corresponds to a codeword design objective, were then provided. It was demonstrated by computer simulation that the precoded UWB system with a reduced-rank codeword outperforms the CPPUWB system with a binary codeword at the cost of a slightly increased feedback overhead, if the channel estimation is accurate enough.

\section{APPENDICES}

\section{A. Proof of Lemma 1}

We first rewrite matrix $\mathbf{R}_{\overline{\mathbf{a}}}$ as

$$
\begin{aligned}
& \mathbf{R}_{\overline{\mathbf{a}}}=\Omega\left[\begin{array}{cccc}
\beta^{2(L-1)} & \alpha \beta^{2 L-3} & \cdots & \alpha \beta^{L-1} \\
\alpha \beta^{2 L-3} & \beta^{2(L-2)} & \cdots & \alpha \beta^{L-2} \\
\vdots & \vdots & \ddots & \vdots \\
\alpha \beta^{L-1} & \alpha \beta^{L-2} & \cdots & 1
\end{array}\right] \\
& =\Omega(1-\alpha)\left[\begin{array}{ccc}
\beta^{2(L-1)} & & \mathbf{0} \\
& \ddots & \\
\mathbf{0} & & 1
\end{array}\right]+\Omega \alpha \mathbf{b b}^{T} \text {, }
\end{aligned}
$$

where

$$
\mathbf{b}=\left[\beta^{L-1}, \cdots, \beta, 1\right]^{T} .
$$

By Theorem 8.1 .8 in [16], the $i$ th eigenvalue $\lambda_{i}$ can be bounded by

$$
\Omega(1-\alpha) \beta^{2 i} \leq \lambda_{i} \leq \Omega(1-\alpha) \beta^{2(i-1)}, \quad 1 \leq i \leq(L-1) .
$$

Next, we will show that the following inequality

$$
\lambda_{i}<\Omega \beta^{2 i}, \quad 1 \leq i \leq(L-1),
$$

holds in our system by the argument of contradiction. We first assume that

$$
\lambda_{i} \geq \Omega \beta^{2 i},
$$

for $1 \leq i \leq(L-1)$. Eq. (39) implies that

$$
\Omega(1-\alpha) \beta^{2(i-1)} \geq \lambda_{i} \geq \Omega \beta^{2 i} .
$$

By substituting $\alpha=\pi / 4$ and $\beta=e^{-\Delta / 2 \Gamma}$ into (40) and performing some simplifications, we get

$$
\frac{\Delta}{\Gamma} \geq-\ln \left(1-\frac{\pi}{4}\right) \approx 1.54,
$$

which suggests that the UWB pulse width is greater than the channel decay time constant. However, this is not true due to the short pulse width of UWB signaling. Therefore, the assumption made is not correct. Thus, we conclude that (38) is true. Finally, since

$\operatorname{tr}\left(\mathbf{R}_{\overline{\mathbf{a}}}\right)=\sum_{i=0}^{L-1} \Omega \beta^{2 i}=\sum_{i=0}^{L-1} \lambda_{i}=\lambda_{0}+\sum_{i=1}^{L-1} \lambda_{i}<\lambda_{0}+\sum_{i=1}^{L-1} \Omega \beta^{2 i}$,

we get $\lambda_{0}>\Omega$.

\section{B. Proof of Proposition 3}

Recall that $\mu_{m}=E\left\{h_{L-1-m}^{2}\right\}=\Omega \beta^{2(L-1-m)}$. Proposition 3 can be proved by showing that

$$
\bar{P}_{s p m}^{(d)}=P \sum_{i=0}^{d-1} \lambda_{i}>P \sum_{m=L-d}^{L-1} \mu_{m}=P \sum_{i=0}^{d-1} \Omega \beta^{2 i}
$$

is true. Note that the maximum peak power generated by the first $d$ columns of $\mathbf{M}$ can be written as

$$
\bar{P}_{\text {spm }}^{(d)}=P \sum_{i=0}^{d-1} \lambda_{i}=P\left(\sum_{i=0}^{L-1} \lambda_{i}-\sum_{j=d}^{L-1} \lambda_{j}\right) .
$$

From Lemma 1, we have the following lower bound for $\bar{P}_{s p m}^{(d)}$ :

$$
\begin{aligned}
\bar{P}_{s p m}^{(d)} & =P\left(\sum_{i=0}^{L-1} \lambda_{i}-\sum_{j=d}^{L-1} \lambda_{j}\right) \\
& >P\left(\sum_{i=0}^{L-1} \Omega \beta^{2 i}-\sum_{j=d}^{L-1} \Omega \beta^{2 j}\right)=P \sum_{i=0}^{d-1} \Omega \beta^{2 i},
\end{aligned}
$$

which is based on the inequality in (38) and

$$
\operatorname{tr}\left\{\mathbf{R}_{\overline{\mathbf{a}}}\right\}=\sum_{i=0}^{L-1} \lambda_{i}=\sum_{i=0}^{L-1} \Omega \beta^{2 i} .
$$

\section{Proof of Proposition 4}

By using (37), the sum of $\lambda_{i}, 1 \leq i \leq L-1$, can be bounded by

$$
\sum_{i=1}^{L-1} \Omega(1-\alpha) \beta^{2 i} \leq \sum_{i=1}^{L-1} \lambda_{i} \leq \sum_{i=1}^{L-1} \Omega(1-\alpha) \beta^{2(i-1)} .
$$

After some manipulations, the above inequality becomes

$$
\Omega(1-\alpha) \frac{1-\beta^{2(L-1)}}{1-\beta^{2}} \beta^{2} \leq \sum_{i=1}^{L-1} \lambda_{i} \leq \Omega(1-\alpha) \frac{1-\beta^{2(L-1)}}{1-\beta^{2}} .
$$

Since

$$
\lambda_{0}+\sum_{i=1}^{L-1} \lambda_{i}=\sum_{i=0}^{L-1} \Omega \beta^{2 i}=\Omega \frac{1-\beta^{2 L}}{1-\beta^{2}},
$$

the signal power, $\lambda_{0}$, due to the use of codeword $\mathbf{c}_{s p m}^{(1)}$ can be bounded by

$$
\begin{aligned}
& \left\{\Omega \frac{1-\beta^{2 L}}{1-\beta^{2}}-\Omega(1-\alpha) \frac{1-\beta^{2(L-1)}}{1-\beta^{2}}\right\} \leq \lambda_{0} \\
& \quad \leq\left\{\Omega \frac{1-\beta^{2 L}}{1-\beta^{2}}-\Omega(1-\alpha) \frac{1-\beta^{2(L-1)}}{1-\beta^{2}} \beta^{2}\right\}
\end{aligned}
$$

If we normalize $\lambda_{0}$ with $\Omega \frac{1-\beta^{2 L}}{1-\beta^{2}}$, which is the average power spread in the channel, (46) becomes

$1-(1-\alpha) \frac{1-\beta^{2(L-1)}}{1-\beta^{2 L}} \leq \tilde{\lambda}_{0} \leq 1-(1-\alpha) \beta^{2} \frac{1-\beta^{2(L-1)}}{1-\beta^{2 L}}$, 
where $\tilde{\lambda}_{0}$ is the normalized power concentrated by $\mathbf{c}_{s p m}^{(1)}$. For $\beta=e^{-\Delta / 2 \Gamma}<1$ and $L \gg 1$, we have

$$
\frac{1-\beta^{2(L-1)}}{1-\beta^{2 L}} \approx 1 \text {. }
$$

Thus, the upper and lower bounds can be approximated by

$$
\begin{gathered}
1-(1-\alpha) \beta^{2} \frac{1-\beta^{2(L-1)}}{1-\beta^{2 L}} \approx 1-(1-\alpha) \beta^{2} \\
=\left(1-\beta^{2}\right)(1-\pi / 4)+\pi / 4
\end{gathered}
$$

and

$$
1-(1-\alpha) \frac{1-\beta^{2(L-1)}}{1-\beta^{2 L}} \approx 1-(1-\alpha)=\alpha=\pi / 4,
$$

respectively. Since $\Delta \ll \Gamma$ is generally true due to an excellent multipath resolution in UWB systems, we have the approximation $\beta=e^{-\Delta / 2 \Gamma} \approx 1$. Then, both the upper and lower bounds are close to $\pi / 4$, which implies that $\tilde{\lambda}_{0} \approx \pi / 4$. It is thus concluded the signal power generated by $\mathbf{c}_{s p m}^{(1)}$ accounts for about $78 \%(\approx \pi / 4)$ of the total power spread in the channel.

\section{REFERENCES}

[1] T. Strohmer, M. Emami, J. Hansen, G. Papanicolaous, and A. J. Paulraj, "Application of time-reversal with MMSE equalization to UWB communications," in Proc. IEEE GLOBECOM'04, Nov. 2004.

[2] K. Usuda, H. Zhang, and M. Nakagawa, "Pre-RAKE performance for pulse based UWB system in a standardized UWB short-range channel," in Proc. IEEE WCNC'04, 2004.

[3] M. Z. Win and R. A. Scholtz, "On the energy capture of ultrawide bandwidth signals in dense multipath environments," IEEE Commun. Lett., vol. 2, no. 9, pp. 245-247, Sep. 1998.

[4] Y.-H. Chang, S.-H. Tsai, X. Yu, and C.-C. J. Kuo, "Ultra-wideband (UWB) transceiver design using channel phase precoding (CPP)," IEEE Trans. Signal Process., vol. 55, no. 7, pp. 3807-3822, 2007.

[5] S. Imada and T. Ohtsuki, "Pre-RAKE diversity combining for UWB systems in IEEE 802.15 UWB multipath channel," in Proc. Joint UWBST \& IWUWBS'04, May 2004.

[6] J. S. Goldstein, I. S. Reed, and L. L. Scharf, "A multistage representation of the Wiener filter based on orthogonal projections," IEEE Trans. Inf. Theory, vol. 44, no. 7, pp. 2943-2959, Nov. 1998.

[7] M. L. Honig and J. S. Goldstein, "Adaptive reduced-rank interference suppression based on the multistage Wiener filter," IEEE Trans. Commun., vol. 50, no. 6, pp. 986-994, 2002.

[8] Y.-L. Chao and R. A. Scholtz, "Weighted correlation receivers for ultra-wideband transmitted reference systems," in Proc. IEEE GLOBECOM'04, Nov. 2004.

[9] IEEE 802.15 high rate alternative PHY task group 3a (TG3a) for wireless personal area networks (WPANs), http://www.ieee802.org/15/pub/TG3a.html.

[10] D. Cassioli, M. Z. Win, and A. F. Molisch, "The ultra-wide bandwidth indoor channel: from statistical model to simulations," IEEE J. Sel. Areas Commun., vol. 20, no. 6, pp. 1247-1257, Aug. 2002.

[11] A. F. Molisch, "Ultrawideband propagation channels-theory, measurement, and modeling," IEEE Trans. Veh. Technol., vol. 54, no. 5, pp. 1528-1545, Sep. 2005.

[12] M. Emami, M. Vu, J. Hansen, A. J. Paulraj, and G. Papanicolaous, "Matched filtering with rate back-off for low complexity communications in very large delay spread channels," in Proc. 38th Asilomar Conference, Nov. 2004.

[13] J. R. Foerster, "Channel modeling sub-committe report final," IEEE P802.15 WPAN P802.15-02/490r1-SG3a, Feb. 2003.

[14] R. A. Horn and C. R. Johnson, Matrix Analysis. Cambridge, UK: Cambridge University Press, 1985.

[15] V. Lottici, A. D'Andrea, and U. Mengali, "Channel estimation for ultrawideband communications," IEEE J. Sel. Areas Commun., vol. 20, no. 9, pp. 1638-1645, Dec. 2002.
[16] G. H. Golub and C. F. V. Loan, Matrix Computations, 3rd ed. Baltimore, MD: Johns Hopkins Press, 1996.

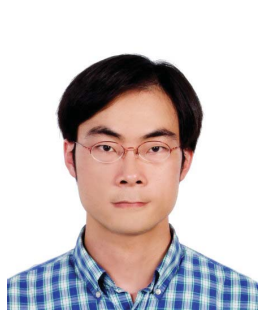

Yu-Hao Chang was born in Taichung, Taiwan, in 1976. He received the B.S. degree in Electrical and Control Engineering from National Chiao Tung University, Hsinchu, Taiwan, in 1998 and the M.S. and $\mathrm{Ph} . \mathrm{D}$. degrees in Electrical Engineering from the University of Southern California (USC) in 2002 and 2007, respectively. Since 2008, he joined MediaTek Inc., Taiwan, and worked on the next generation mobile communication standards, such as IEEE $802.16 \mathrm{~m}$ and 3GPP LTE-Advanced.

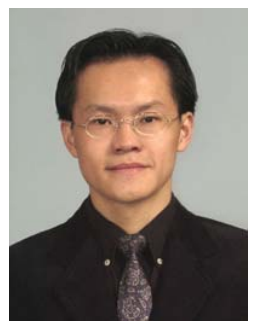

Shang-Ho (Lawrence) Tsai received the Ph.D. degree in Electrical Engineering from the University of Southern California, USA, in Aug. 2005. From June 1999 to July 2002, he was with the Silicon Integrated Systems Corp. (SiS), where he participated the VLSI design for DMT-ADSL systems. From Sep. 2005 to Jan. 2007, he was with the MediaTek Inc. (MTK) and participated the VLSI design for MIMO-OFDM systems. Since Feb. 2007, he joined the Department of Electrical and Control Engineering (now Department of Electrical Engineering) at the National Chiao Tung University where he is now an assistant professor. His research interests include signal processing for communications, particularly the areas of OFDM as well as MIMO systems. He is also interested in Ultra Wideband (UWB) and the VLSI design related to above topics. He was awarded a government scholarship for overseas study from the Ministry of Education, Taiwan, in 2002-2005.

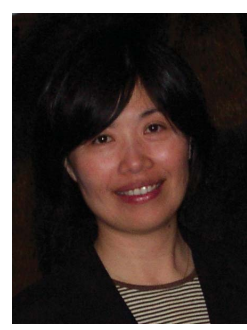

Xiaoli Yu received the Ph.D. degree in Electrical Engineering from the University of Southern California, Los Angeles, CA in 1991. Her research interests are in the areas of applying statistical signal processing and communication and information theory to wireless telecommunications systems and biological systems.

In 1992, she was with the Space Computer Corp in Santa Monica, CA as a research scientist. From 1993 to 1997 , she was a system engineer and then a research instructor both at the Molecular Imaging Science Center, the Radiology Department of Keck School of Medicine in the University of Southern California. Since 1998, she has been an assistant professor of Departments of Radiology and Electrical Engineering in the University of Southern California. Dr. Yu received the 1999-2000 IEE ClarkeGriffiths Memorial Premium for the best paper in the IEE PROCEEDINGS ON RADAR, SONAR, AND NAVIGATION.

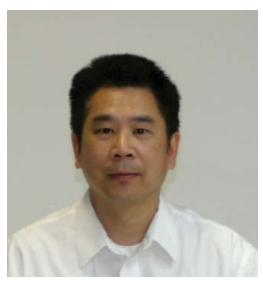

C.-C. Jay Kuo received the B.S. degree from the National Taiwan University, Taipei, in 1980 and the M.S. and Ph.D. degrees from the Massachusetts Institute of Technology, Cambridge, in 1985 and 1987, respectively, all in Electrical Engineering. Dr. Kuo was Computational and Applied Mathematics (CAM) Research Assistant Professor in the Department of Mathematics at the University of California, Los Angeles, from October 1987 to December 1988. Since January 1989, he has been with the University of Southern California, where he is currently Professor of Electrical Engineering and Computer Science and Director of the Signal and Image Processing Institute. His research interests are in the areas of digital signal and image processing, multimedia compression, communication and networking technologies. He is a co-author of about 160 journal papers, 780 conference papers and 10 books.

Dr. Kuo is a Fellow of IEEE and SPIE. He is Editor-in-Chief for the JOURNAL OF Visual COMMUNiCATION AND IMAGE REPRESENTATION, and Editor for the JOURNAL OF INFORMATION SCIENCE AND ENGINEERING, LNCS Transactions on Data Hiding and Multimedia SeCUrity and the EURASIP Journal of APPlied Signal Processing. Dr. Kuo received the National Science Foundation Young Investigator Award (NYI) and Presidential Faculty Fellow (PFF) Award in 1992 and 1993, respectively. 